\title{
Research on power control system of inspection robot based on wavelet transform
}

\author{
GUAN Hong-mei ${ }^{1}$,LIU Yue ${ }^{2}$ \\ \{dawda985@163.com ${ }^{1}$, tosn614@163.com²\}
}

(1. Department of Automation, College of Humanities Information Changchun University of Technology, Changchun 130122, China;

2.Department of Automation, College of Humanities Information Changchun University of Technology, Changchun 130122, China)

\begin{abstract}
Due to the poor stability of the traditional power control system, inspection control errors will occur during the inspection process. Therefore, a new type of power supply control system for inspection robots was designed based on wavelet transform, and the drive hardware circuit and control hardware circuit of inspection robots were redesigned in hardware design to ensure the stability of hardware operation. In the software part, by extracting and checking the structure and functional characteristics of the robot, setting the system control parameters based on the wavelet transform, and configuring the system communication parameters, in order to enhance the stability of the system operation and realize the complete design of power control. The test results show that, compared with the traditional power control system, the designed system has better stability performance and stronger patrol robot control ability. The designed power control system can be applied to the patrol robot control.
\end{abstract}

Keywords: Wavelet transform;Patrol robot;Power control system

\section{Introduction}

There are many shortcomings in the traditional manual inspection method. High labor intensity, low work efficiency, scattered detection quality, and single means; under severe weather conditions such as thunderstorms, manual inspections have greater safety risks and cannot be inspected in time; traditional video surveillance systems are restricted by various conditions There is a large blind spot in surveillance, and it is difficult to truly meet the requirements of all-round coverage of video surveillance. At the same time, due to the complexity of the system, the large number of cameras, and the large workload of installation and wiring, the failure rate is high and maintenance is difficult. With the promotion of smart substation and unattended substation modes, higher requirements are placed on the inspection of substations, and a more advanced and intelligent inspection mode is urgently needed.

With the rapid development of special robot technology in recent years, inspection robot technology that replaces manual inspection has become the focus of attention at home and abroad. Checking robots involves various disciplines and needs to be studied in combination with actual conditions. Only by combining the previous experience and actual investigation can the design of the main body of the transmission line robot and the obstacle avoidance strategy be completed, and the corresponding control system be designed to achieve. The line 
is easy to meet the emergency, so it is necessary to monitor the data and status of the robot in real time during the inspection of the robot, and transmit the image data to the ground, which can be used to remotely control the robot through the ground, and the remote operating system evaluates the patrol of the transmission line Environment, find the inspection status of the transmission line, perform simple processing, etc. [1]. However, the traditional design method has insufficient control stability, so it is necessary to propose a new design scheme to correct the traditional design method. In the redesign, the design of the inspection robot power control system is based on the wavelet transform theory as the basic design idea. The hardware circuit of the power supply control system improves the stability of the control system through continuous wavelet transform, thereby achieving the reliability of the inspection robot. This article involves the system to provide a powerful guarantee for improving the inspection work of the inspection robot.

\section{Design of hardware circuit of power control system of patrol robot}

\subsection{Design of driving circuit for inspection robot}

The driving circuit of the circuit patrol motion control system mainly consists of three parts:(2) the obstacle removal system that controls the robot to remove high-voltage line obstacles;(3) robotic arm system to control the movement of robot manipulator.The routing system controlling the robot walking is mainly used to control the forward and backward functions of the robot on the line. The driving control circuit is shown in figure 1.

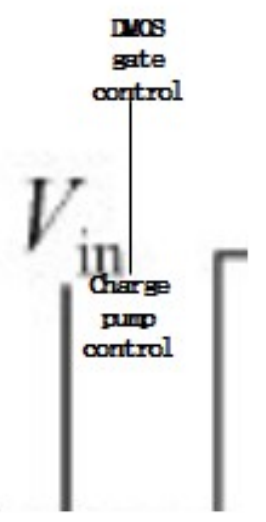

Fig. 1. Inspection robot drive circuit.

The main control chip TMS320F28335 sends the control signal of high and low level to the circuit. When the GPI081 pin sends a low voltage to the circuit, ${ }^{R_{1}}$ and ${ }^{R_{3}}$ divide the voltage, ${ }^{R_{3}}$ divide the voltage of $1 \mathrm{~V}$, the transistor ${ }^{L_{1}}$ realizes conduction, the relay circuit conducts conduction, and the $24 \mathrm{~V}$ voltage is connected to the stepper motor driver. Then the main control chip is used to input the PWM signal and the high and low level signal to drive 
the stepper motor forward and backward, so as to realize the forward and backward control of the robot.When GPI081 pin sends a high level (3.3v) to the circuit, ${ }^{R_{1}}$ and $R_{3}$ divide the voltage, ${ }^{R_{3}}$ divide the $0.34 \mathrm{v}$ voltage, the transistor ${ }^{L_{1}}$ turns off, and the stepper motor stops $[2]$.

In order to reduce the control error of the line inspection robot, the single power supply mode is adopted, and the $24 \mathrm{~V}$ battery is used as the power control system hardware of the whole robot.However, since multiple chips need to be powered, $24 \mathrm{~V}$ to SV power supply circuit composed of $1 \mathrm{~m} 2576 \mathrm{~s}-5.0$ voltage regulator chip is adopted here to solve this problem. The circuit shown in figure 2 is a schematic diagram of SV voltage stabilizing circuit.

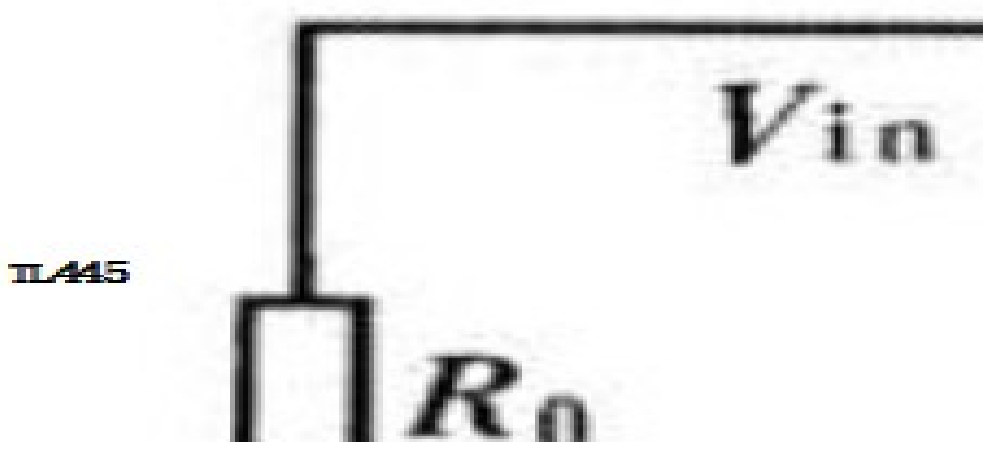

Fig. 2. Regulating circuit.

The voltage regulator chip used in the voltage regulator circuit is $\operatorname{lm} 2 \mathrm{~s} 76 \mathrm{~s}-\mathrm{s} .0$, which belongs to the step-down switching voltage regulator, has very small voltage adjustment rate and current adjustment rate, and has the load driving capacity of $3 \mathrm{~A}$, which can replace the general three-terminal linear voltage regulator, fully reduce the area of the heat sink, and even do not use the heat sink under some application conditions.At the same time, the chip is relatively simple and has fewer peripheral components, built-in frequency compensation circuit and fixed frequency oscillator, as well as built-in overcurrent protection circuit and overheat protection circuit.Under the conditions of specified input voltage and output load, the input voltage of $\operatorname{lm} 2 \mathrm{~s} 76 \mathrm{~s}-\mathrm{s} .0$ reaches the fixed voltage output of $36 \mathrm{~V}$ and $5 \mathrm{~V}$, and the error range of the output voltage is $\pm 14 \%$. The oscillation frequency error range of the oscillator is $10 \%$. The typical standby current is $50^{\circ} \mathrm{A}$, thus realizing the hardware design of the driving circuit of the patrol robot [3].

\subsection{Electric control circuit design of inspection robot}

Based on the hardware design of the driving circuit of the inspection robot, the control circuit hardware of the power control system of the inspection robot is designed.Known inspection robot movement, mainly rely on the power control system board send action instruction, so as to complete the corresponding action, at the same time control system board needs to be real-time display robot servo system signal acquisition system, the working state of the robot were collected including robots working voltage, working current, robot power. The block diagram of the control circuit hardware design of the power control system is 
shown in figure 3 below.

Since the power control system of the inspection robot is the key control center of the control drive system, the main control chip of the power control system of the inspection robot based on wavelet transform is MSP430F437.

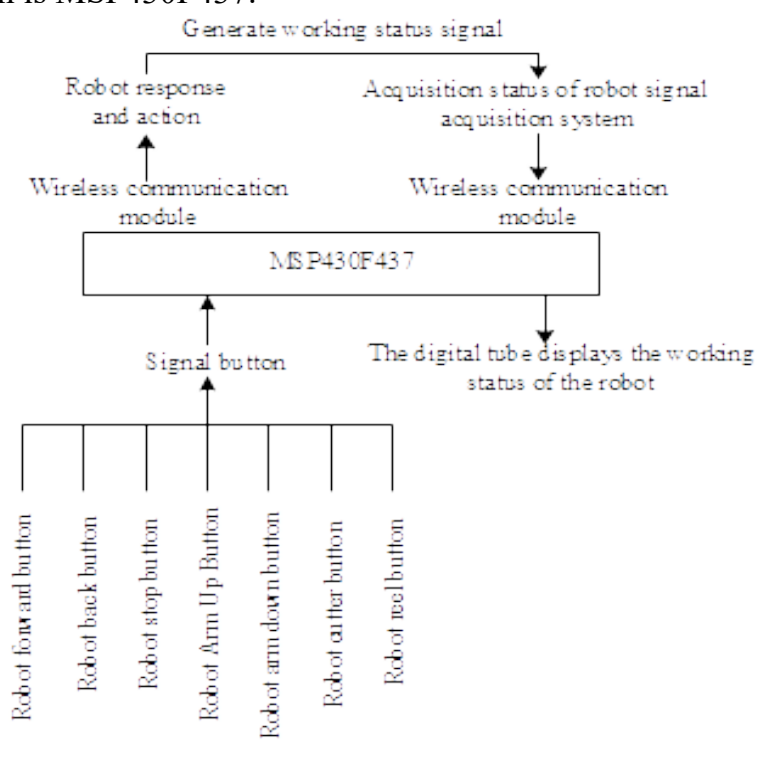

Fig. 3. The control circuit design diagram of the inspection robot.

According to the process described in the figure 3 , the control port circuit of the robot control board is designed.In the control panel of the known robot, the button used for the signal sending button is a waterproof self-locking button with LED lamp, and the button port circuit is shown in figure 4 [4].

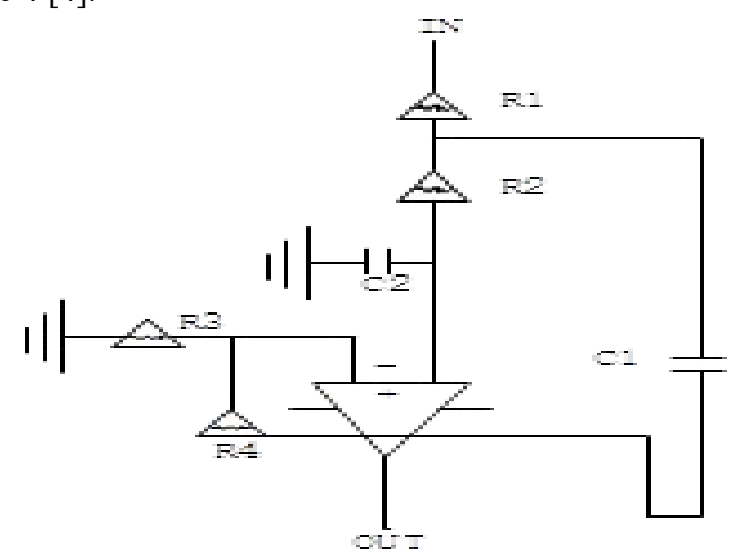

Fig. 4. Button port circuit.

When the self-locking button is pressed, the first pin and the third pin in the figure are connected to the first pin through a diode. When the self-locking button is released, the first 
pin and the third pin are disconnected. Two control chip pins with $1 \mathrm{~K}$ electricity connected to the first pin through a diode are set as high level.Then the hardware of the numerical display circuit of the control board of the inspection robot is designed.The design of the circuit is for real-time robot working status in the process of movement, through the timely display of robot inspection work, to achieve timely adjustment of the control system. The digital tube display circuit is shown in figure 5 .

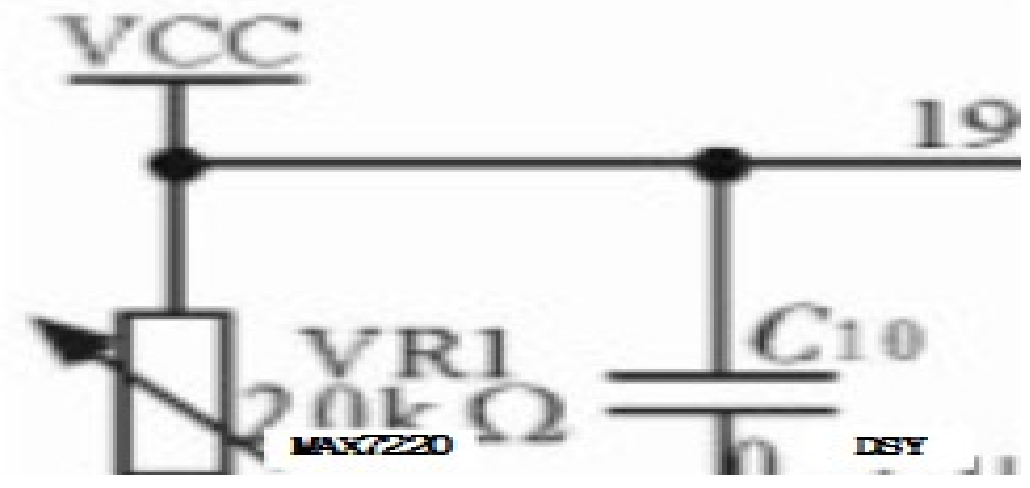

Fig. 5. Digital tube display circuit.

According to the design of drive circuit hardware and control circuit hardware, it is convenient for the application of wavelet transform technology in power control system.

\section{Design control system software based on wavelet transform}

On the basis of the circuit design, the control system software is redesigned according to the wavelet transform theory and application technology.

\subsection{Extract the dynamic characteristics of the inspection robot}

The inspection robot consists of many parts, including support columns; Inspection platform, lift structure, beam, etc.Because the structure parameters of the robot during the inspection are not fixed, it is necessary to analyze the dynamic characteristics of the inspection robot in order to optimize the design of the power control system software.Figure 6 is the schematic diagram of the inspection robot in the visual direction. 


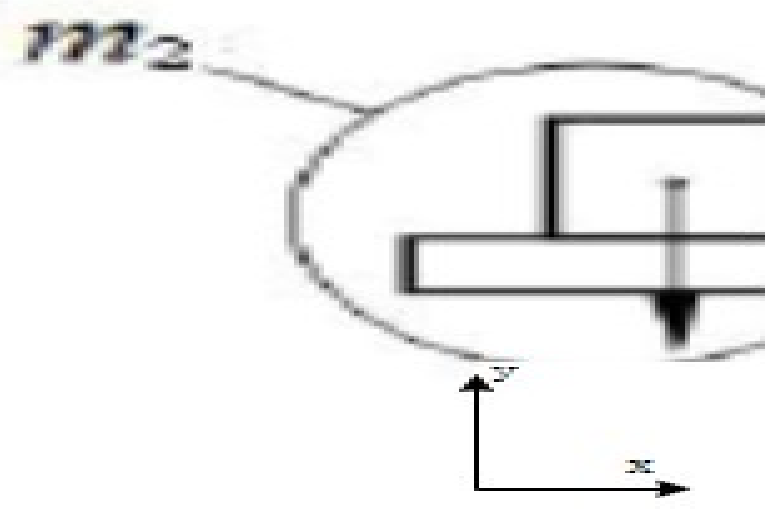

Fig. 6. Inspection robot structure diagram.

According to the parameter information in the figure 6 , the inertial dynamic value of the robot is calculated as follows:

$$
F_{s}=\frac{C_{z}^{3} H_{s}}{12}-\frac{c_{s}^{3}-h_{s}}{12}
$$

Where: ${ }^{F_{z}}$ represents the inertial dynamic value of size ${ }^{s} ; C_{s}$ represents the size of the robot base; ${ }^{C_{2}}$ represents the top size of the robot inspection device; ${ }_{3}$ represents the horizontal height of the robot; ${ }^{h}$ represents the distance from the base of the robot to the ground.It is known that under different working intensivities, robots will have different motion modes due to different working properties and working environments, and the deformation occurring in the plane XOY [5] has the greatest impact on their working characteristics. When the robot is stationary, its static deflection is basically unchanged, but when starting and braking, the deflection of the robot column increases with the increase of acceleration, and the deflection of the column has a great relationship with the horizontal acceleration. Therefore, the deflection of the robot column is calculated as:

$$
\sigma=\sigma_{g}+\sigma_{f}+\sigma_{q}
$$

Where: $\sigma_{g}$ represents the deflection of the column tip caused by gravity of each mass element; ${ }^{\sigma}$ represents the deflection of the column tip caused by the inertial force of each mass element; $\sigma_{q}$ represents the deflection at the top of the column caused by the inertial force evenly distributed by the column itself.According to the results obtained in formula (1) and formula (2), the dynamic characteristics of the inspection robot are obtained as follows:

$$
k=\sum \frac{x_{i} y_{i} \varphi_{i} g}{F_{z}}+\frac{\sigma}{4 E I} \sum+\varphi_{i} z_{i}(\nu h-c)+\frac{\sigma}{6 E I}
$$

Where: ${ }^{k}$ represents the dynamic characteristic value of the robot; ${ }^{x_{i}}, y_{i}$ and $z_{i}$ 
represent the node coordinates of the ${ }^{i}$ mobile joints of the inspection robot; ${ }^{\varphi_{i}}$ represents the load capacity value of the robot; $\stackrel{g}{ }$ represents the bending moment of each mass element to the axis of the column; $v$ is the acceleration of gravity; $h$ represents the height of the robot; $c$ represents the acceleration in the walking direction; $E I$ represents the force modulus [6].According to the above formula, the dynamic characteristics of the inspection robot are extracted.

\subsection{The system control parameters are set based on wavelet}

According to the obtained dynamic characteristics of the robot, the system control parameters based on wavelet transform are set. The setting process of such parameters is shown in figure 7 below.

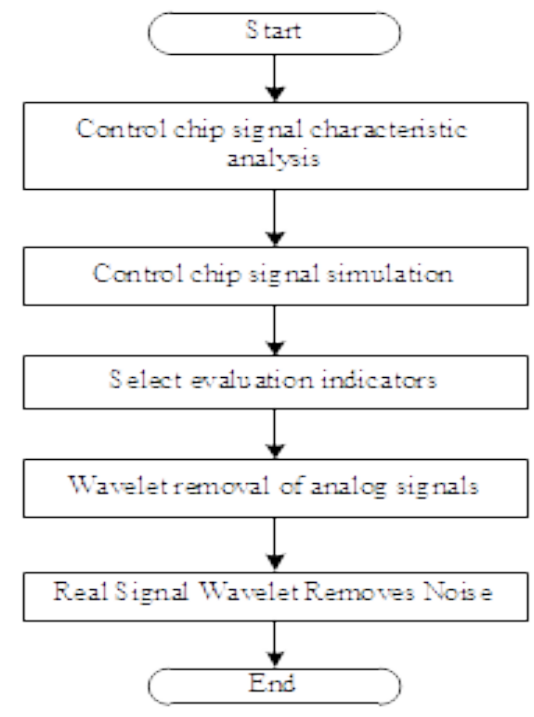

Fig. 7. System control parameter setting process.

According to the figure 7 , the calculation condition of the wavelet function is set as follows: $\tau(u) \in Y^{-2}(R)$, its Fourier transform is $\tau(v)$, and it satisfies $\tau(0)=0$, then $\tau(u)$ can generate the family of functions through expansion and translation $\left\{\tau_{a, b}\right\}$, and the relation is:

$$
\tau_{a, b}(u)=|a|^{-\frac{1}{2}} \tau\left(\frac{u-b}{a}\right)
$$

Where $a$ represents the scale factor, $b$ represents the translation factor, and $a, b \in R$, $a \neq 0 ; \tau$ represents the base wavelet or the mother wavelet [7].According to the continuous wavelet transform results of the square integrable function obtained from the above equation, the transformation equation of the integrable function $h(u) \in Y^{-2}(R)$ is:

$$
(W, h)(a, b)=\frac{1}{\sqrt{a}} \int_{-\infty}^{+\infty} h(u) \tau^{\mathrm{Y}}\left(\frac{u-b}{a}\right) d u=\left[h, \tau_{a, b}\right]
$$


Where: $\left(W_{:}, h\right)(a, b)$ represents the continuous wavelet transform coefficient; $Y$ is the conjugate. $a>0, b$ can be positive or negative in practice. Since each parameter is a continuous variable, the control structure of the system by parameters is set according to the linear, time-shift and scale characteristics of the continuous wavelet, as shown in figure 8 below.

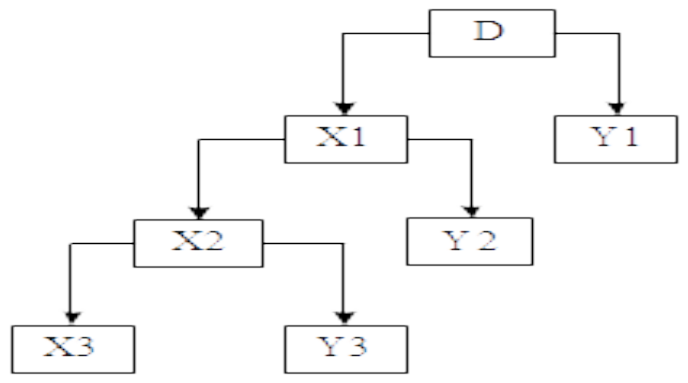

Fig. 8. Parameter control structure diagram.

In the figure $8, \mathrm{D}$ represents the original signal, and $\mathrm{X} 1, \mathrm{X} 2$ and $\mathrm{X} 3$ represent the parameters of surface data control structure in the three stages.Y1, Y2 and Y3 represent the hidden control structure parameters of the three stages, and set the control parameters [8].

3.3 Configuration of communication parameters to achieve the power control system design

To complete the communication between control system software, but also to configure the communication parameters.According to the communication control mode of the power control system of the inspection robot, the communication protocol code of the control algorithm is adjusted, and the adjusted parameter values are shown in table 1 below [9].

Table 1. Results of communication parameter adjustment.

\begin{tabular}{ccc}
\hline Parameter name & Dynamic value & Static value \\
\hline Communication control & 2074 & 2000 \\
Frequency setting & 2085 & 2001 \\
Inverter status & 3863 & 30001 \\
Operating frequency & 3485 & 3001 \\
Set frequency & 3095 & 3003 \\
The output voltage & 2374 & 2001 \\
Output current & 2099 & 2000 \\
\hline
\end{tabular}

According to the communication change parameters in table 1 above, the demo program of the power control system is set, and the schematic diagram of the delay program is shown in figure 9 below. 


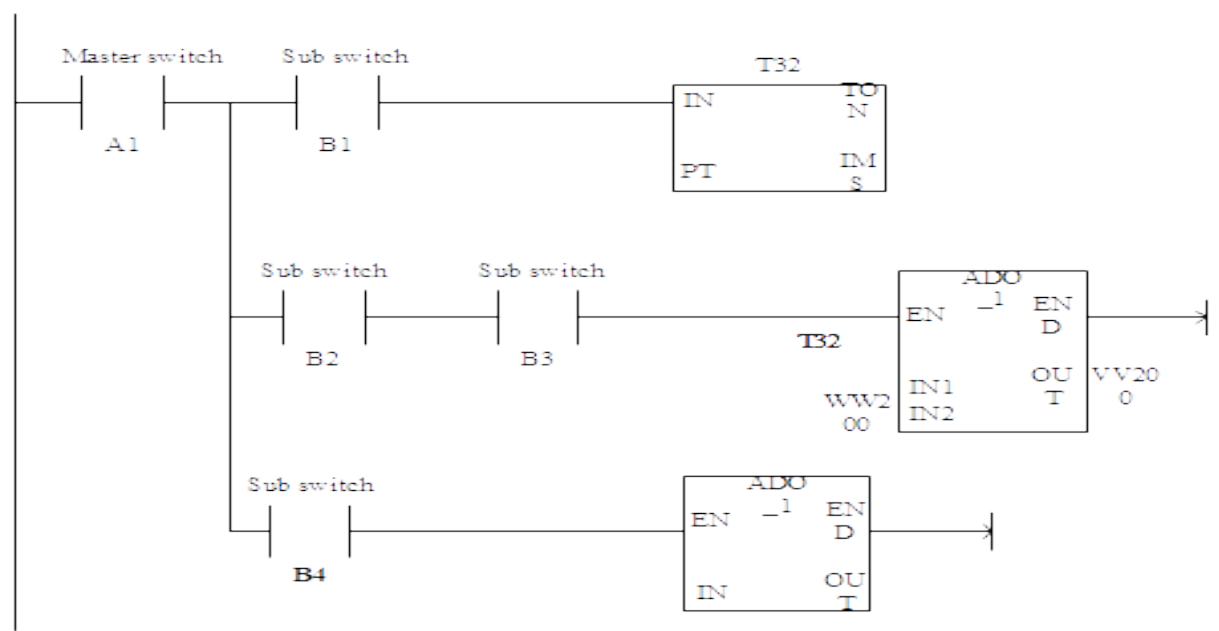

Fig. 9. Communication delay program.

According to the delay program set in the figure 9, the response sensitivity of the inspection robot is controlled to ensure that all movement of the robot can meet the requirements of inspection. Thus, the electric control system of the inspection robot based on wavelet transform is designed [10].

\section{Experimental analysis}

In order to verify the effectiveness of the research in this paper, a comparative test experiment was put forward. The designed power control system and the traditional designed power control system were put into the use of patrol robots. According to the use effect of patrol robots, specific experimental test conclusions were drawn.

Two inspection robots with the same model and configuration were selected as experimental test objects, and two power control systems were loaded into the robot. The physical picture of the inspection robot is selected, as shown in Figure 10. 


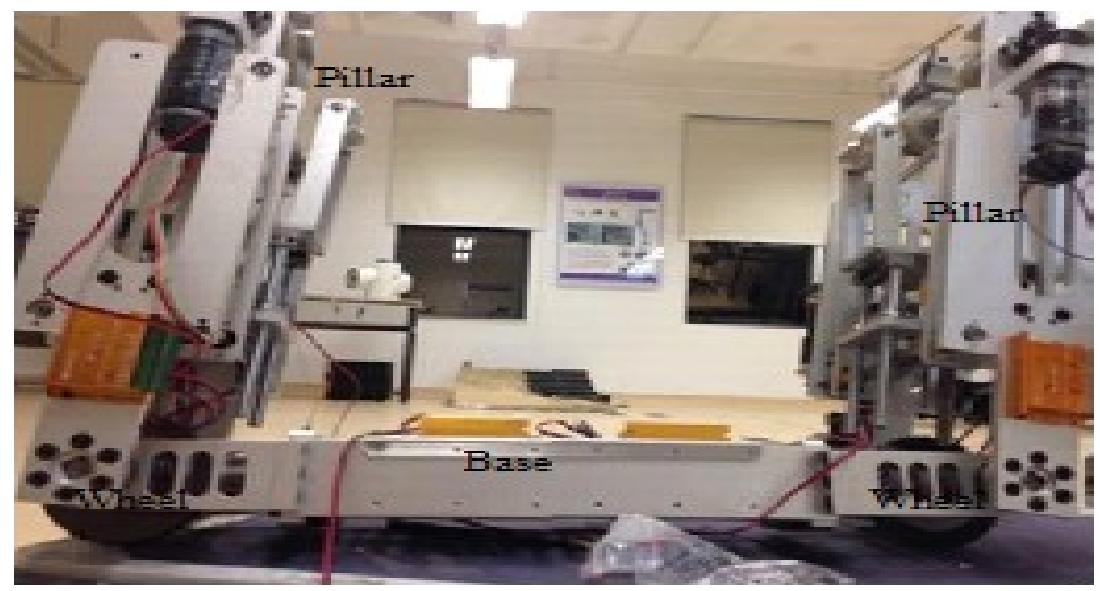

Fig. 10. Patrol robot prototype.

The experimental test platform is mainly composed of the following parts: the experimental support is used to simulate the power line, and the robot can detect and cross over the simple obstacles on the top; The pressure measuring unit is set up to measure the pressure output of the clamping and pressing mechanism when the robot goes over obstacles.At the same time, remote sensing connection will be established between the inspection robot, key control box and computer to ensure the continuity of the test system.After completing the above operations, the test capability of the experimental test system is tested. The following table 2 is the test system operation parameter values obtained by the test.

Table 2. Test the parameters of the system running smoothly.

\begin{tabular}{ccc}
\hline Testing time & Stability parameter & deviation \\
\hline hh & 0.99 & 0 \\
$3 \mathrm{~h}$ & 0.99 & 0 \\
$4 \mathrm{~h}$ & 0.99 & 0 \\
$5 \mathrm{~h}$ & 0.99 & 0 \\
$6 \mathrm{~h}$ & 0.99 & 0 \\
$7 \mathrm{~h}$ & 0.99 & 0 \\
$8 \mathrm{~h}$ & 0.98 & 0.01 \\
$9 \mathrm{~h}$ & 0.99 & 0 \\
$10 \mathrm{~h}$ & 0.99 & 0 \\
$11 \mathrm{~h}$ & 0.98 & 0.01 \\
$12 \mathrm{~h}$ & 0.98 & 0.01 \\
$13 \mathrm{~h}$ & 0.98 & 0.01 \\
$14 \mathrm{~h}$ & 0.98 & 0.01 \\
$15 \mathrm{~h}$ & 0.97 & 0.02 \\
$16 \mathrm{~h}$ & 0.97 & 0.02 \\
$17 \mathrm{~h}$ & 0.97 & 0.02 \\
$18 \mathrm{~h}$ & 0.97 & 0.02
\end{tabular}




$\begin{array}{lll}19 \mathrm{~h} & 0.96 & 0.03 \\ 20 \mathrm{~h} & 0.96 & 0.03 \\ 21 \mathrm{~h} & 0.95 & 0.04 \\ 22 \mathrm{~h} & 0.95 & 0.04 \\ 23 \mathrm{~h} & 0.95 & 0.04 \\ 24 \mathrm{~h} & 0.94 & 0.05\end{array}$

According to the above table, within 24 hours, the deviation between the stability index of the test system and the required value of standard stability is within 0.05 , so it can be known that the test result of the system is reliable and the experiment can be started.

In this experiment, the test results of the inspection robot control system based on wavelet transform were taken as the experimental group.The test results of the electric power control system of the patrol robot designed under the traditional design method were taken as the control group, as shown in figure 11 below, that is, the experimental test comparison results.

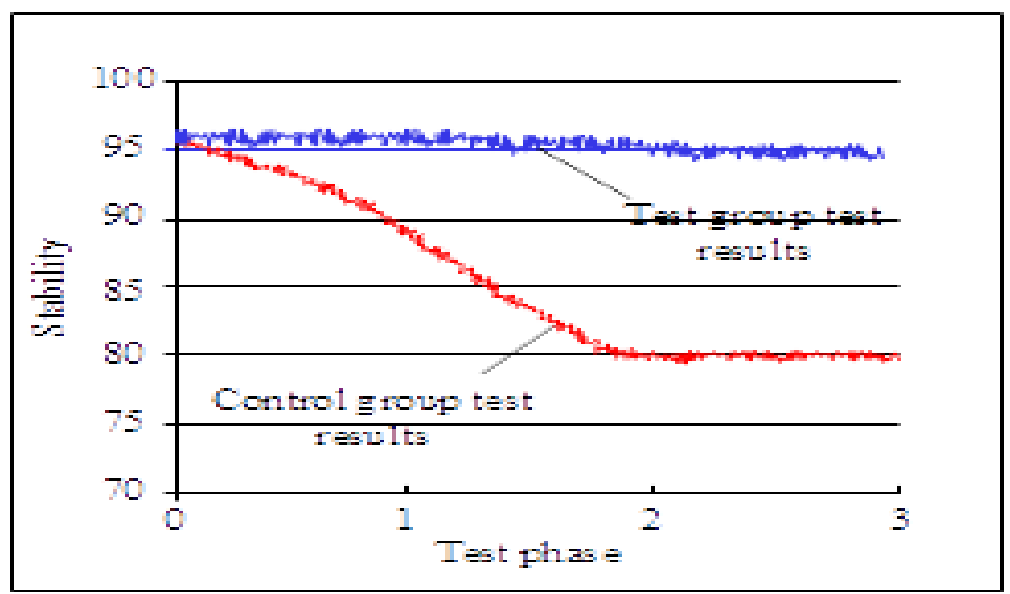

Fig. 11. Experimental test comparison results.

According to the test results in the figure 11 , the stability of the power control system designed based on wavelet transform decreased by $0.5 \%$ in the third stage, and the calculated average stability was about $95.13 \%$.However, the stability of the power control system designed by the traditional method began to decline in the first stage, and by the third stage, the stability degree was already around $80 \%$, and the decline degree was about $15.86 \%$.It can be seen that the designed power control system is more suitable for the daily control requirements of patrol robot.

\section{Conclusion}

The design of electric control system based on wavelet transform, the drawbacks of the existing in the traditional control system as the breakthrough point, take full advantage of wavelet transform concept, to better drive hardware circuit and communication circuit of hardware support, has realized the speed inspection robot more moderate, stability, strong electrical control system design, for use in the inspection robot in the future development, to 
provide reliable technical support.But the design of the control system, in the hardware circuit part did not explain in detail, driving circuit hardware, as well as all the hardware circuits in the communication circuit, the future research can be aimed at this problem, make detailed data description.

\section{References}

[1] XUE Yang, ZHANG Xiaoyu, JIANG Tianbo, etal. Double-mode Control of Inspection Robots Based on Visual Navigation[J]. Control Engineering of China, 2018,25(11):1982-1987.

[2] YANG Lin, MA Hongwei, WANG Chuanwei, etal. Intelligent navigation and control of campus inspection robot[J]. Journal of Xi'an University of Science and Technology,2018,38(06):1013-1020.

[3] WANG Zhifang, FU Xingjian, SHEN Jie, etal. Robust adaptive fault tolerate control for stability control of electric patrol survey UAV[J].Engineering Journal of Wuhan University,2018,51(07):646653.

[4] SONG Yu, WEN Xi, LI Yan, etal. A Telepresence Control System for Mobile Robot Based on Google Glass[J].Control Engineering of China,2018,25(06):1077-1085.

[5] TAN Xiaoheng, CHU Guoxing, ZHANG Xuejing, etal. Modulation recognition algorithm based on high-order cumulants and wavelet transform[J]. Systems Engineering and Electronics, 2018,40(01):171-177.

[6] DAI Dangdang, WANG Xianpei, LONG Jiachuan, etal. De-noising Method of Ultra-high Frequency Partial Discharge Signal Based on Improved Protrugram and Wavelet Transform[J]. High Voltage Engineering, 2018,44(11):3577-3586.

[7] ZHANG Anlong, LI Yan, HUANG Fuquan, etal. Adaptive protection and self-healing control method of distribution network based on dynamic topology analysis[J]. Power System Protection and Control, 2019,47(11):111-117.

[8] DING Feng, XIAO Yangting, ZHANG Shaohua.Study on Energy Management Control System and All-digital Simulation of Ship Integrated Power System [J]. Ship Engineering, 2018,40(05):4651.

[9] HOU Junjie, FAN Yanfang, ZHONG Xian, etal. Research on high resistance grounding time domain equation based distance protection for wind power accessed power system[J].Power System Protection and Control,2018,46(01):122-128.

[10] Cai Dongsheng, Huang Qi, Li Jian, etal. Research on control method of power system with timedelays based on improved IQC algorithm[J].Electrical Measurement \& Instrumentation, 2019,56(01):15-21. 